\title{
Centrifugal Pathways Protect Hearing Sensitivity at the Cochlea in Noisy Environments That Exacerbate the Damage Induced by Loud Sound
}

\author{
Ramesh Rajan \\ Department of Physiology, Monash University, Monash, Victoria 3800, Australia
}

\begin{abstract}
Loud sounds damage the cochlea, the auditory receptor organ, reducing hearing sensitivity. Previous studies demonstrate that the centrifugal olivocochlear pathways can moderately reduce these temporary threshold shifts (TTSs), protecting the cochlea. This effect involves only the olivocochlear pathway component known as the crossed medial olivocochlear system pathway, originating from the contralateral brainstem and terminating on outer hair cells in the cochlea. Here I demonstrate that even moderate noise backgrounds can significantly exacerbate the cochlear TTSs induced by loud tones, but this is prevented because in such conditions there is additional activation of uncrossed olivocochlear pathways, enhancing protection of co-
\end{abstract}

chlear hearing sensitivity. Activation of the uncrossed pathways differs from that of the crossed pathway in that it is achieved only in noise backgrounds but can then be obtained under monaural conditions of loud tone and background noise. In contrast, activation of the crossed pathway is achieved only by binaural loud tones and is not further enhanced by background noise. Thus, conjoint activation of both crossed and uncrossed efferent pathways can occur in noise backgrounds to powerfully protect the cochlea under conditions similar to those encountered naturally by humans.

Key words: cochlea; olivocochlear efferents; loud sounds; hearing damage; noise backgrounds; protection; TTS
An early manifestation of the damaging effects of loud sounds is a temporary hearing desensitization [temporary threshold shifts (TTSs)] that occurs without any morphological damage to cochlear structures or only variable damage (Robertson and Johnstone, 1980; Robertson et al., 1980; Robertson, 1982; Tilney et al., 1982; Liberman and Dodds, 1987; Gao et al., 1992; Harding et al., 1992; Dew et al., 1993; Borg et al., 1995). It is generally accepted (Cody and Russell, 1986; Liberman et al., 1986; Patuzzi et al., 1989; Cooper and Rhode, 1992; Patuzzi, 1992; Ruggero et al., 1993) that TTSs is primarily attributable to effects on the outer hair cells (OHCs) of the cochlea, whose local actions in transduction are critical for normal hearing sensitivity and frequency selectivity (Harrison and Evans, 1977; Dallos and Harris, 1978; Liberman and Kiang, 1978; Robertson and Johnstone, 1979; Schmeidt et al., 1980; Cody and Russell, 1986; Liberman et al., 1986; Patuzzi and Robertson, 1988; Cody, 1992).

The mammalian cochlea receives a dual-component efferent innervation (Guinan et al., 1983; Warr et al., 1986). The lateral olivocochlear system (LOCS), almost exclusively from only the ipsilateral lateral superior olivary nucleus, terminates on dendrites of afferent neurons. The medial olivocochlear system (MOCS), from ipsilateral [uncrossed MOCS (UMOCS)] and contralateral [crossed MOCS (CMOCS)] periolivary nuclei, terminates on OHCs. Physiological activation of the MOCS by binaural stimulation can reduce TTSs induced by loud tones (Cody and Johnstone, 1982; Rajan and Johnstone, 1988; Rajan, 1992, 1995a,b, 1996). Only the CMOCS pathway is involved, and lesioning only this pathway prevents the effect (Rajan and Johnstone, 1988; Rajan, 1995b).

The efferent effect on TTSs to damaging tones shows features consistent with a protective system: protection is graded to TTSs (Rajan and Johnstone, 1988; Rajan, 1992, 1995b), occurs to any loud tone, but is evoked most easily by tones most readily damaging the cochlea (Rajan, 1995b), and occurs to each of successive loud tones (Rajan, 1996). This has lead to suggestions (Rajan, 1992;

\footnotetext{
Received April 18, 2000; revised May 31, 2000; accepted June 13, 2000.

This work was supported by Grant 970505 from the National Health and Medical Research Council of Australia.

Correspondence should be addressed to Dr. Rajan at the above address. E-mail: ramesh.rajan@med.monash.edu.au.

Copyright (C) 2000 Society for Neuroscience $0270-6474 / 00 / 206684-10 \$ 15.00 / 0$
}

Brown et al., 1998) that cochlear centrifugal pathways have an important functional role at high sound levels and to interest in determining if they were involved in other forms of protection from loud sound (Kujawa and Liberman, 1997; Zheng et al., 1997a), including from permanent damage (Handrock and Zeisberg, 1982; Liberman and Gao, 1995; Zheng et al., 1997b). However, because classical electrically elicited efferent effects are strongest on cochlear responses at low sound levels and decline by high levels (Galambos, 1956; Weiderhold, 1986), the functional value of this protection is yet to be widely accepted and it is sometimes described as an epiphenomenon (Borg et al., 1995) to a primary, yet unknown, role. Here I demonstrate that in background noise, there is conjoint activation of crossed and uncrossed efferent pathways to powerfully protect (by almost $30 \mathrm{~dB}$ ) from loud sound whose effects would otherwise be exacerbated by the noise. This compelling evidence for a functional role for efferent protection from loud tone-induced damage has direct relevance to human susceptibility to loud sounds in noisy workplace or recreational environments.

\section{MATERIALS AND METHODS}

Animal treatment and measurement of hearing sensitivity. Procedures involved in animal treatment and measuring cochlear hearing sensitivity have been detailed elsewhere (Rajan et al., 1991; Rajan, 1995a). In brief, adult cats weighing between 3 and $6 \mathrm{~kg}$ were tested under procedures approved by the Monash University Standing Committee on Ethics in Animal Experimentation and conforming to guidelines of the National Health and Medical Research Council of Australia. Cats were anesthetized $(60 \mathrm{mg} / \mathrm{kg})$ and maintained with continuous intravenous infusion of Nembutal at 2-3 $\mathrm{mg} \cdot \mathrm{kg}^{-1} \cdot \mathrm{hr}^{-1}$. Depth of anesthesia was monitored through continuous recording of rectal temperature, ECG, and EMG activity from forearm muscles and by regular hourly checks of the response to strong noxious pinching of the forepaw and of the presence of pupillary dilatation and absence of corneal reflexes. The output from the ECG/EMG electrodes was displayed on an oscilloscope and fed into a speaker for continuous monitoring of the cat's condition and depth of anesthesia. Body temperature was maintained at $37.5 \pm 0.5^{\circ} \mathrm{C}$ by a thermostatically controlled warming blanket, regulated by feedback from a rectal probe. Cats were tracheostomized and artificially ventilated on room air. Tidal volume was determined from normogram cat respiratory data. Respiratory rate was set between 20 and 25 breaths/min depending on the cat's size.

Surgery (Rajan et al., 1991) was performed to implant stainless steel electrodes against the round window membrane of both cochleas to measure cochlear hearing sensitivity bilaterally. Hearing sensitivity was assessed by measuring thresholds for the compound action potential (CAP) of the auditory nerve at frequencies from 1 to $40 \mathrm{kHz}$ (Rajan et al., 1991; 
Table 1. Test groups and experimental conditions of laterality of loud tone exposure (loud tone always at $13 \mathrm{kHz}, 100 \mathrm{~dB} \mathrm{SPL}$ for $10 \mathrm{~min}$ ) and background to exposure

\begin{tabular}{|c|c|c|c|}
\hline Group & $\begin{array}{l}\text { Status of efferent pathways } \\
\text { [All pathways intact/All pathways cut/ } \\
\text { Only crossed pathways cut (leaving intact } \\
\text { uncrossed pathways)] }\end{array}$ & $\begin{array}{l}\text { Loud tone } \\
\text { exposure laterality }\end{array}$ & Background to the tone exposure \\
\hline \multicolumn{4}{|c|}{ Groups to establish the basic protective effects of cochlear efferents } \\
\hline Silence 1 & All pathways intact & Monaural & Silence \\
\hline Silence 2 & All pathways intact & Binaural & Silence \\
\hline Silence 3 & $\begin{array}{l}\text { Ear 1: all pathways intact } \\
\text { Ear 2: all pathways cut }\end{array}$ & Binaural & Silence \\
\hline Silence 4 & $\begin{array}{l}\text { Ear 1: only crossed pathways cut } \\
\text { Ear 2: only crossed pathways cut }\end{array}$ & Binaural & Silence \\
\hline \multicolumn{4}{|c|}{ Groups to establish that cochlear efferents provide extra protection in noise backgrounds } \\
\hline Noise 1 & $\begin{array}{l}\text { Ear 1: all pathways intact } \\
\text { Ear 2: all pathways cut }\end{array}$ & Binaural & Binaural Noise@40 dB SPL \\
\hline Noise 2 & $\begin{array}{l}\text { Ear 1: all pathways intact } \\
\text { Ear 2: all pathways cut }\end{array}$ & Binaural & Binaural Noise@60 dB SPL \\
\hline Noise 3 & $\begin{array}{l}\text { Ear 1: all pathways intact } \\
\text { Ear 2: all pathways cut }\end{array}$ & Binaural & Binaural Noise@80 dB SPL \\
\hline \multicolumn{4}{|c|}{ Groups to establish the role of uncrossed pathways in extra protection by cochlear efferents in noise background } \\
\hline Noise 4 & $\begin{array}{l}\text { Ear 1: only crossed pathways cut } \\
\text { Ear 2: only crossed pathways cut }\end{array}$ & Binaural & Binaural Noise@80 dB SPL \\
\hline Noise 5 & $\begin{array}{l}\text { Ear 1: only crossed pathways cut } \\
\text { Ear 2: all pathways cut }\end{array}$ & Binaural & Binaural Noise@80 dB SPL \\
\hline Noise 6 & All pathways intact & Monaural & Monaural Noise@80 dB SPL \\
\hline Noise 7 & $\begin{array}{l}\text { Ear 1: only crossed pathways cut } \\
\text { Ear 2: all pathways cut }\end{array}$ & $\begin{array}{l}\text { Monaural } \\
\text { Monaural }\end{array}$ & $\begin{array}{l}\text { Monaural Noise@80 dB SPL } \\
\text { Monaural Noise @80 dB SPL }\end{array}$ \\
\hline
\end{tabular}

Rajan, 1995a). Thresholds were compared to normative data (Rajan et al., 1991; Rajan, 1995a), and only animals with normal hearing sensitivity bilaterally from 1 to $40 \mathrm{kHz}$ were used.

Tones and noise stimuli to each ear were generated independently by one of four channels of a digital synthesis system, gated under computer control and passed through separate computer-controlled attenuators, before feeding into one of four channels of an electronic mixer box. The mixer was used to manually switch delivery of stimuli to each ear as desired. Crosstalk between different channels of the mixer box was more than $-100 \mathrm{~dB}$ up to $10 \mathrm{kHz},-100 \mathrm{~dB}$ from $10-20 \mathrm{kHz}$, and declined thereafter to $-95 \mathrm{~dB}$ at $40 \mathrm{kHz}$. Two output channels from the mixer box separately fed sound to one of two Sennheiser HD 535 speakers, each in specially designed housing leading out to a sound delivery tube placed in one external auditory meatus (Rajan et al., 1991).

Surgical inactivation of efferent pathways. Inactivation of various components of the cochlear efferent pathways was made using surgical lesions at the floor of the fourth ventricle, after removing the overlying cerebellum (Rajan, 1995a). Because of the coursing of the efferent fibers, it is possible from this brainstem location to lesion all efferent pathways to one or both cochleas, or crossed pathways to both cochleas (Warren and Liberman, 1989), but not the crossed pathway to only one cochlea or only the uncrossed pathways to a cochlea. Thus, lesions were made to either totally de-efferent only one cochlea (Rajan, 1995a,b), lesion only the crossed efferent pathways bilaterally (Rajan, 1995a,b), or combine these manipulations to cut crossed pathways to both cochleas and all efferent pathways to one cochlea, leaving only uncrossed pathways intact to only one cochlea.

To totally de-efferent only one cochlea, a lesion was made $1.5-2 \mathrm{~mm}$ lateral of the midline and on the brainstem side ipsilateral to the cochlea to be de-efferented. To cut only crossed pathways (bilaterally), the lesion was made exactly at the midline. To cut crossed pathways to both cochleas and all efferent pathways to one cochlea, two lesions were placed as a combination of the previous two lesion types. The first lesion was at the midline to cut crossed pathways bilaterally and a second lesion placed 1.5-2 $\mathrm{mm}$ lateral of the midline to cut all efferent pathways to only one cochlea. Lesions were always 6- to 8-mm-long, extending around the location of the facial colliculi that were identifiable on the floor of the fourth ventricle. Postmortem histological analysis, occasionally combined with histochemical staining for acetylcholine esterase (which stains the efferent pathways), was used to confirm the location of the cuts (Warren and Liberman, 1989; Rajan, 1995a).

In all animals with lesions placed in the brainstem, the CAP audiogram was measured before and after placing any lesions. The heart rate, ECG waveform, and body temperature were also noted before the lesion and rechecked immediately after the lesion.
Traumatic loud sound exposures and measurement of cochlear desensitization. The traumatic loud tone used to cause TTSs was at $13 \mathrm{kHz}$ and was presented at $100 \mathrm{~dB}$ sound pressure level (SPL) continuously for $15 \mathrm{~min}$. This frequency was chosen as it is from within the most sensitive part of the cat's CAP audiogram (Rajan et al., 1991) and, of particular relevance to this study, frequencies from this region cause hearing damage more easily than do other frequencies (Rajan, 1995b), as well as more readily activate the previously described (Rajan, 1995b) protective effects of the crossed efferent pathways.

With monaural testing the loud tone was delivered to only the test ear; the output to the non-test ear was blocked off by turning off the mixer box switch to that ear and by turning off, through computer control, that output channel from the stimulus synthesis system. With binaural testing the loud tone was delivered simultaneously and concurrently to both ears. In testing the effects of noise backgrounds, continuous noise $(0.5-40 \mathrm{kHz})$ was switched on $3 \mathrm{sec}$ before the loud tone and remained on for the duration of the tone. Tone and noise were switched off simultaneously by computer control after $15 \mathrm{~min}$ of loud tone (and noise) exposure.

Cats were assigned to groups exposed to the loud tone in a background of silence or of noise. Groups exposed to the tone in a background of silence were: Silence $1(n=6$ cats $)$ and Silence $2(n=6)$, exposed monaurally and binaurally, respectively, without any lesions to cochlear efferent pathways; Silence $3(n=5)$, exposed binaurally after a lesion to cut all cochlear efferent pathways unilaterally; and Silence 4, exposed binaurally after lesions of only crossed pathways bilaterally $(n=2)$. Noise groups were exposed to the loud tone in a background of noise. In Noise $1(n=4)$, the noise level was $40 \mathrm{~dB}$ SPL, in Noise $2(n=7)$ at $60 \mathrm{~dB}$ SPL, and in Noise $3(n=6)$, Noise $4(n=5)$, Noise $5(n=6)$, Noise $6(n=6)$, and Noise $7(n=5)$ at $80 \mathrm{~dB}$ SPL. Groups Noise $1-5$ were exposed to the tone and noise binaurally, Noise 1-3 after unilateral total de-efferentation, Noise 4 after a lesion of only crossed pathways (bilaterally), and Noise 5 after lesions to cut crossed pathways bilaterally as well as all efferent pathways (i.e., the remaining uncrossed pathways) to only one side. Groups Noise 6 and 7 were exposed monaurally to the tone in noise, in Noise 6 with no lesions to cochlear efferent pathways and in Noise 7 after the same two types of lesions as in Noise 5. In Noise 7 the crossed de-efferented ears were tested first and later, after data collection from these ears, the totally de-efferented ears were tested, also monaurally. The experimental conditions in each group are summarized in Table 1.

A further group (group "Noise-alone") was tested with monaural noise by itself at $80 \mathrm{~dB}$ SPL either with no lesions to efferent pathways $(n=2)$ or after unilateral de-efferentation $(n=8)$. To ensure that any noise effects would not be missed, thresholds were measured from $15 \mathrm{sec}$ after the 

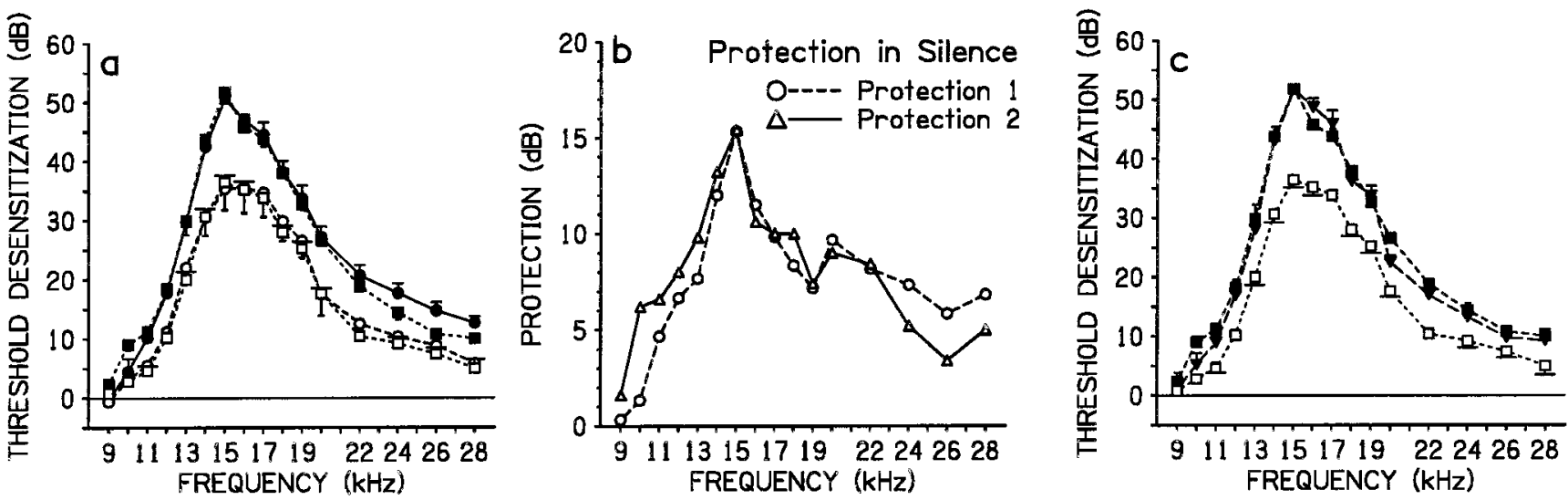

Figure 1. In a background of silence the crossed efferent pathway is activated by binaural, but not monaural, stimulation to protect the cochlea from loud sound-induced TTSs. $a$, $c$, Loud sound-induced TTSs of frequency-specific thresholds for the auditory nerve CAP. Data are mean data (error bars indicate $\mathrm{SE}$ ) from groups or treatment conditions exposed to a loud tone in a background of silence. In $a$, binaural tonal exposure in group Silence 2 (open circles and dotted line) produced lower TTSs than did monaural exposure in group Silence 1 (closed circles and full line); and in Silence 3, binaural tonal exposure after unilateral lesion of all efferent pathways produced TTSs in de-efferented ears in Silence 3 (closed squares and long dashed line) similar to those in Silence 1, and TTSs in efferent-intact ears in Silence 3 (open squares and short dashed line) similar to those in Silence 2. In $c$, group Silence 4 (closed inverted triangles and long dashed line) was exposed binaurally after bilateral lesions of only the crossed efferent pathways; this resulted in TTSs in these crossed de-efferented ears similar to those in the totally de-efferented ears in Silence 3 (closed squares and short dashed line) and larger than in efferent-intact ears in Silence 3 (open squares and short dashed line). $b$, Protection of the cochlea in treatment conditions from $a$ : Protection 1 was the difference in mean TTSs in Silence 1 and those in Silence 2; Protection 2 was the difference in mean frequency-specific TTSs in totally de-efferented ears and in efferent-intact ears of group Silence 3 .

noise-alone exposure, much earlier than the post-test measurement time in all other groups exposed to the loud tone, as detailed below.

In all groups with loud tone exposure, CAP thresholds were measured 5 min after the end of the loud tone at frequencies from 7 to $30 \mathrm{kHz}$, in a constant (but not linear) order. It took $\sim 2.5 \mathrm{~min}$ to measure thresholds from 9 to $28 \mathrm{kHz}$ bilaterally. Frequency-specific TTSs were calculated as the difference between the pre-loud tone and post-loud tone thresholds. Comparisons between groups or treatments were comparisons between TTSs at corresponding frequencies. Two-way repeated measures ANOVAs were used to compare effects between different experimental conditions. If the ANOVA revealed a significant difference between conditions, generally with a significant interaction term between experimental condition and frequency, unpaired Student's $t$ tests were used to compare threshold losses at corresponding frequencies in the two conditions.

\section{RESULTS}

\section{Basic protective effects of the crossed olivocochlear pathway on TTSs induced by loud tones in a background of silence}

For the standard loud tone here (at $13 \mathrm{kHz}, 100 \mathrm{~dB}$ SPL for 15 min), I first confirmed previous demonstrations (Cody and Johnstone, 1982; Rajan and Johnstone, 1988; Rajan, 1995a,b) using other loud tones (at different frequencies, and for different durations) that in a background of silence, binaural, but not monaural, loud sound could activate the crossed efferent pathway to reduce loud tone-induced TTSs. These results will be compared to the effects of the loud sound in noise backgrounds.

In a background of silence, the standard loud tone applied monaurally (group Silence 1; Fig. $1 a$ ) produced TTSs $>25 \mathrm{~dB}$ at frequencies from 13 to $20 \mathrm{kHz}$. Damage decreased outside this range, more rapidly at lower than higher frequencies. Maximum threshold shift of $\sim 50 \mathrm{~dB}$ occurred at $15 \mathrm{kHz}$. Binaural exposure to the same tone (group Silence 2; Fig. 1a) resulted in significantly smaller TTSs from 11 to $28 \mathrm{kHz}$ (Table $2 A$ ). Reductions in TTSs (i.e., protection; Fig. $1 b$, Protection 1 ) were generally graded to TTSs occurring with monaural exposure (i.e., to the corresponding-frequency data in group Silence 1), and the largest mean reduction of $\sim 15 \mathrm{~dB}$ was at $15 \mathrm{kHz}$. These effects of binaural versus monaural exposure are identical to those reported previously for other loud sounds applied in silence (Cody and Johnstone, 1982; Rajan and Johnstone, 1988; Rajan, 1995a,b).

Surgical inactivation of cochlear efferent pathways confirmed they were responsible for protection in the binaural condition. In group Silence 3, after totally de-efferenting only one cochlea the loud tone was applied binaurally; from 10 to $28 \mathrm{kHz}$ TTSs in de-efferented ears (Fig. 1a) were significantly larger than in efferent-intact ears (Table 2B). Threshold shifts in the latter ears were always similar to corresponding-frequency TTSs from efferent-intact binaurally exposed group Silence 2 (Table 2B), whereas TTSs in de-efferented Silence 3 ears were similar to those in the monaurally exposed efferent-intact group Silence 1 (Table $2 B$ ). Thus, in silence, binaural loud sound activates cochlear efferents to protect, and monaural loud sound does not. Given this, the protection in Silence 3 (difference between TTSs in de-efferented and efferent-intact ears; Fig. 1b, Protection 2) was identical to the protection seen when comparing the binaurally exposed efferentintact group Silence 2 against the monaurally exposed efferentintact group Silence 1 (Fig. 1b, Protection 1). In both comparisons, maximum protection was at $15 \mathrm{kHz}$ and was $15 \mathrm{~dB}$ reduction from a peak threshold shift of $\sim 50 \mathrm{~dB}$.

This protection involved only the crossed efferent pathway, as shown by lesioning only this pathway (bilaterally) in Silence 4 before binaural application of the loud tone. The identical treatment in the two ears in each animal resulted in bilaterally similar TTSs; data from corresponding frequencies from all ears were therefore pooled. Threshold shifts in these crossed de-efferented ears were similar (Fig. 1c) to those in totally de-efferented ears of the binaurally exposed Silence 3 (Table $2 C$ ) but were significantly larger from 11 to $24 \mathrm{kHz}$ (Table $2 C$ ) than in efferent-intact ears in Silence 3. So, although uncrossed efferent pathways were intact in Silence 4, they did not reduce TTSs, confirming that cochlear protection gained with binaural loud tones in silence is attributable to only the crossed pathway.

\section{There is enhanced protection of hearing sensitivity by cochlear efferent pathways in non-damaging noise backgrounds that can exacerbate loud sound-induced TTSs}

The effects of a noise background to the loud tone on TTSs were first tested in groups in which one cochlea was totally de-efferented. The lesion was followed by binaural exposure to the loud tone in a noise background. Results are compared to results in group Silence 3 , in which the same lesion type was followed by binaural tone exposure in a background of silence.

Depending on intensity, noise backgrounds exacerbated TTSs in de-efferented ears (Fig. 2a). The exacerbation was most pro- 


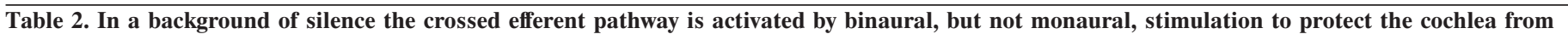
loud sound-induced TTSs

Comparison groups/treatments ${ }^{a}$

ANOVA $F$ and $p$ values (Interaction $=$ group $\times$ frequency interaction)
Frequency range with significant differences ${ }^{d}$ and parameters of significance

A. Binaural exposure to a loud tone in silence results in lower TTS compared to monaural exposure

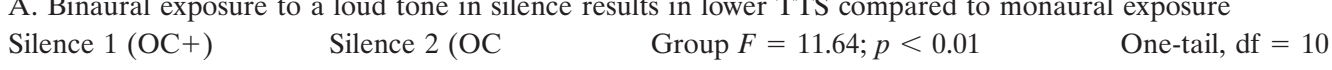

$11-28 \mathrm{kHz} ; t$ always $>1.98 ; p$ generally $<0.01$

Monaural exposure $\quad$ Binaural exposure $\quad$ Interaction $F=3.55 ; p<0.001$

B. Cochlear protection with binaural loud tone exposure in silence is attributable to cochlear efferents that are activated by binaural but not monaural exposure
Silence $3 \mathrm{OC}-$
Silence 3 OC+
Group $F=195.6 ; p<0.001$
One-tail, df $=8$
$10-28 \mathrm{kHz} ; t$ always $>2.6 ; p$ generally $<0.001$

Binaural exposure

Binaural exposure

Interaction $F=7.88 ; p<0.001$

Silence 3 OC-

Binaural exposure

Silence $1(\mathrm{OC}+)$

Group $F=0.07 ; p=0.8$

Monaural exposure

Interaction $F=1.2 ; p=0.26$

Silence $3 \mathrm{OC}+$

Silence 2 OC+

Group $F=0.122 ; p=0.74$

Binaural exposure

Binaural exposure

Interaction $F=0.22 ; p=1$

C. Cochlear protection with binaural exposure in silence is attributable only to the crossed efferent pathway

Silence $4(\mathrm{COC}-) \quad$ Silence $3 \mathrm{OC}-\quad$ Group $F=4.62 ; p=0.07$

Binaural exposure $\quad$ Binaural exposure $\quad$ Interaction $F=1.52 ; p=0.1$

Silence $4(\mathrm{COC}-) \quad$ Silence $3 \mathrm{OC}+\quad$ Group $F=200.3 ; p<0.001 \quad$ One-tail, df $=7$

$11-24 \mathrm{kHz} ; t$ always $>2.33 ; p$

Binaural exposure $\quad$ Binaural exposure $\quad$ Interaction $F=8.09 ; p<0.001$

generally $<0.001$

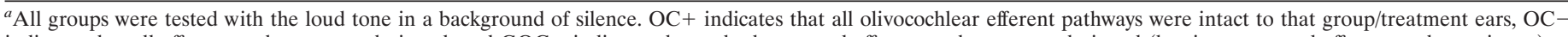

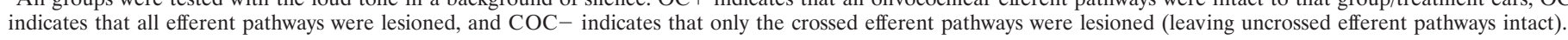

${ }^{b} t$ tests were performed when the ANOVA indicated significant differences.

${ }^{c}$ Degrees of freedom.

${ }^{d}$ Frequencies for which $t$ values were significant at $\alpha=0.05$ (i.e., $p$ at least $<0.05$ ).
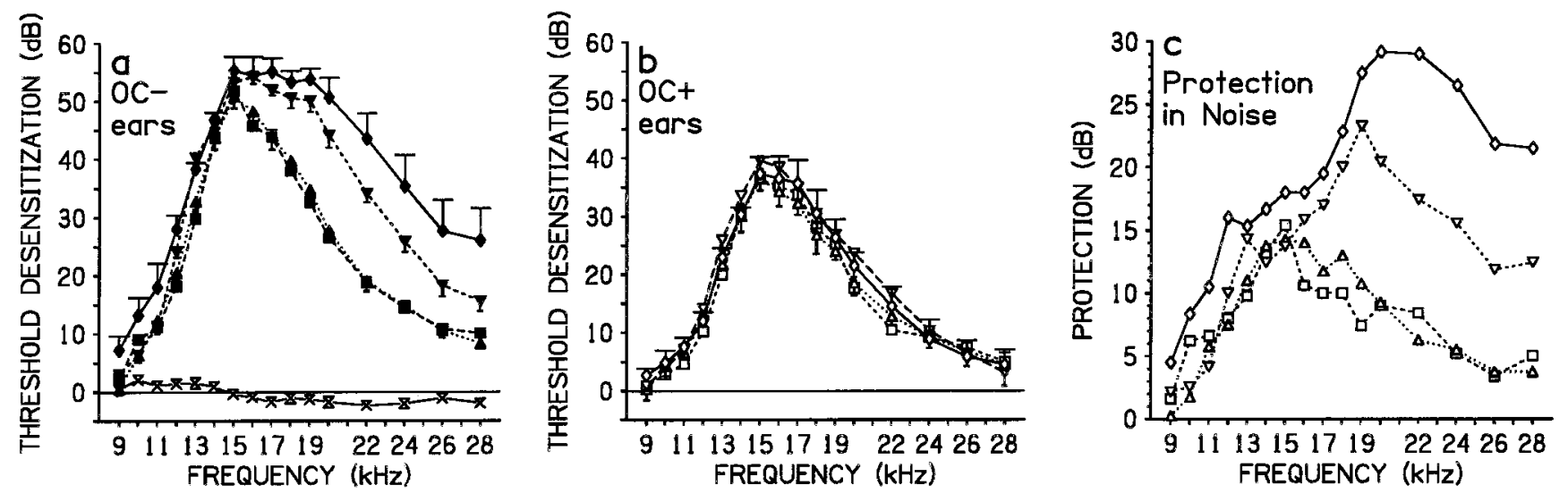

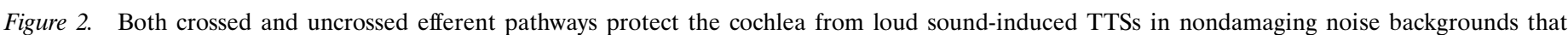

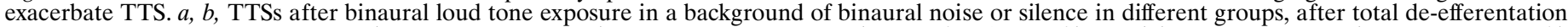

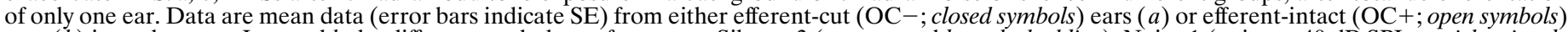

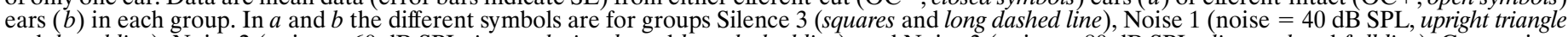

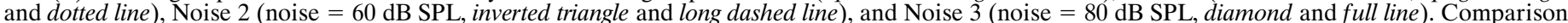

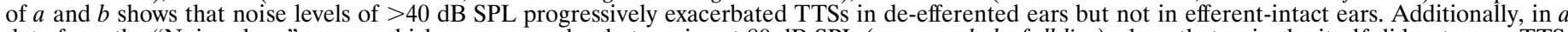

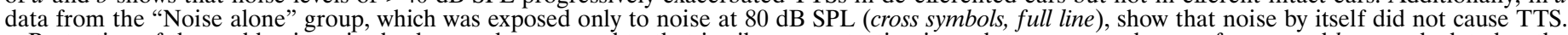

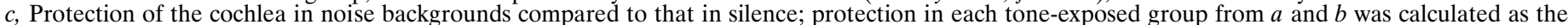

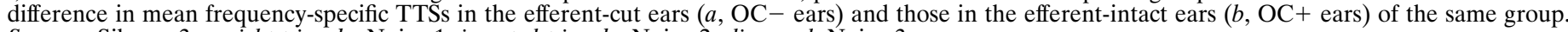
Squares, Silence 3; upright triangle, Noise 1; inverted triangle, Noise 2; diamond, Noise 3.

nounced at frequencies $>15 \mathrm{kHz}$, which was the frequency most affected by the tone in a background of silence. With noise at $40 \mathrm{~dB}$ SPL (group Noise 1) there was no exacerbation of TTSs: frequency-specific TTSs in de-efferented ears were similar to those in de-efferented ears of Silence 3 (Table $3 A$ ). With noise at $60 \mathrm{~dB}$ SPL (Noise 2) or $80 \mathrm{~dB}$ SPL (Noise 3 ) de-efferented ears suffered significantly larger tone-induced TTSs at 12,13 , and from 16 to 28 $\mathrm{kHz}$ than de-efferented ears of Silence 3 (Table $3 A$ ). Comparing the noise groups, increasing noise intensity from 40 to $60 \mathrm{~dB}$ SPL caused significantly greater TTSs mainly from 16 to $28 \mathrm{kHz}$ (Table $3 A$ ), and increasing intensity from 60 to $80 \mathrm{~dB}$ SPL further significantly exacerbated TTSs mainly from 22 to $28 \mathrm{kHz}$ (Table $3 A$ ).

In contrast to de-efferented ears, in efferent-intact ears (Fig. 2b) background noise did not increase TTSs above those occurring in efferent-intact ears to the loud tone in a background of silence. Threshold shifts in each group with a noise background (Noise 1, 


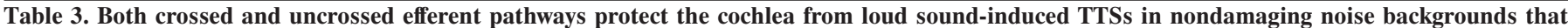
can exacerbate the sound-induced TTS

Comparison groups/treatments

ANOVA $F$ and $p$ values (Interaction $=$ group $\times$ frequency interaction)
Frequency range with significant differences ${ }^{d}$ and parameters of significance

A. In ears with non-functional efferent pathways, noise backgrounds exacerbate loud tone-induced TTS, depending on noise intensity

Silence $3 \mathrm{OC}-$

Noise $1 \mathrm{OC}-$

(noise $=40 \mathrm{~dB}$ SPL)

Silence 3 OC-

Noise 2 OC-

(noise $=60 \mathrm{~dB}$ SPL)

Silence 3 OC-

Noise 3 OC-

(noise $=80 \mathrm{~dB}$ SPL)

Noise 1 OC-

Noise 2 OC-

(noise $=40 \mathrm{~dB}$ SPL)

(noise $=60 \mathrm{~dB}$ SPL)

Noise 2 OC-

Noise 3 OC-

$($ noise $=60 \mathrm{~dB}$ SPL) $\quad$ (noise $=80 \mathrm{~dB}$ SPL) $\quad$ Interaction $F=1.73 ; p<0.05$

Group $F=0.33 ; p=0.59$

Interaction $F=0.74 ; p=0.76$

Group $F=131, p<0.001$

Two-tail, df $=9$

Interaction $F=8.02 ; p<0.001$

Group $F=22.69 ; p<0.001$

Two-tail, df $=8$

Interaction $F=4.92 ; p<0.001$

Group $F=104.3 ; p<0.001$

One-tail, df $=8$

Interaction $F=4.37 ; p<0.001$

Group $F=5.91 ; p<0.05$

One-tail, df $=11$

10, 22-28 kHz; $t$ always $>1.9 ; p$ $<0.05$

B. In efferent-intact ears, noise backgrounds do not exacerbate the TTS caused by a loud tone

$\begin{array}{lll}\text { Silence } 3 \mathrm{OC}+ & \begin{array}{l}\text { Noise } 1 \mathrm{OC}+ \\ \text { (noise }=40 \mathrm{~dB} \text { SPL) }\end{array} & \begin{array}{l}\text { Group } F=0.33 ; p=0.59 \\ \text { Interaction } F=0.74 ; p=0.74\end{array} \\ \text { Silence 3 OC }+ & \begin{array}{l}\text { Noise } 2 \mathrm{OC}+ \\ \text { (noise }=60 \mathrm{~dB} \text { SPL) }\end{array} & \begin{array}{l}\text { Interaction } F=0.99 ; p=0.47 \\ \text { Silence 3 OC+ }\end{array} \\ & \begin{array}{l}\text { Noise } 3 \mathrm{OC}+ \\ \text { (noise }=80 \mathrm{~dB} \text { SPL) }\end{array} & \text { Group } F=0.49 ; p=0.5 \\ & \text { Interaction } F=0.403 ; p=0.4\end{array}$

C. The cochlear protection from loud sound-induced TTS in noise backgrounds is attributable to both crossed and uncrossed efferent pathways

\begin{tabular}{|c|c|c|c|c|}
\hline Noise $4(\mathrm{COC}-)$ & Noise $3 \mathrm{OC}-$ & Group $F=34.22 ; p<0.001$ & One-tail, df $=14$ & $\begin{array}{l}9-28 \mathrm{kHz} ; t \text { always }>2.04 ; p \\
\quad \text { generally }<0.005\end{array}$ \\
\hline
\end{tabular}

$($ noise $=80 \mathrm{~dB}$ SPL) $\quad$ (noise $=80 \mathrm{~dB}$ SPL)

Interaction $F=8.1 ; p<0.001$

Noise $4(\mathrm{COC}-) \quad$ Noise $3 \mathrm{OC}+$

Group $F=7.02 ; p<0.02$

One-tail df $=12$

12-18 kHz; $t$ always $>2.1 ; p$ always $<0.05$

(noise $=80 \mathrm{~dB}$ SPL)

(noise $=80 \mathrm{~dB}$ SPL)

Interaction $F=2.08 ; p<0.01$

Noise $5 \mathrm{COC}-\quad$ Noise $5 \mathrm{OC}+$

Group $F=1093 ; p<0.001$

One-tail, df $=4$

9-28 kHz; $t$ always $>2.7$; $p$ generally $<0.0005$

(noise $=80 \mathrm{~dB}$ SPL)

(noise $=80 \mathrm{~dB}$ SPL)

Interaction $F=25.44 ; p<0.001$

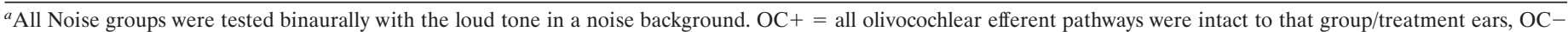
$=$ all efferent pathways were lesioned, and $\mathrm{COC}-=$ only the crossed efferent pathways lesioned (leaving uncrossed efferent pathways intact).

${ }^{b} t$ tests were performed when the ANOVA indicated significant differences.

${ }^{c}$ Degrees of freedom.

${ }^{d}$ Frequencies for which $t$ values were significant at $\alpha=0.05$ (i.e., $p$ at least $<0.05$ ).

2, and 3) were similar (Table $3 B$ ) to TTSs in efferent-intact ears in group Silence 3 exposed to the binaural tone in a background of silence, as well as similar between the three noise groups ( $p$ always $>0.05$, generally $>0.3$ ).

Noise by itself at $80 \mathrm{~dB}$ SPL did not cause any TTSs in deefferented or efferent-intact ears. Data from these conditions were pooled and are illustrated in Figure $2 a$. Noise only induced small and nonsystematic threshold changes, either of increased sensitivity or desensitization, within the $2-3 \mathrm{~dB}$ variability for threshold measurements with the technique used here (Rajan et al., 1991; Rajan, 1995a).

Because higher-level noise backgrounds progressively exacerbated loud tone-induced TTSs only in de-efferented ears, the difference between TTSs in de-efferented and efferent-intact ears (i.e., the protection in efferent-intact ears) increased with increasing noise level, particularly at $>15 \mathrm{kHz}$. The protection in noise backgrounds is illustrated in Figure $2 c$ and compared to that in group Silence 3 , in which unilateral de-efferentation was followed by binaural tone exposure in a background of silence. In the latter case protection followed the pattern of TTS. Mean protection $>10$ $\mathrm{dB}$ occurred from 13 to $18 \mathrm{kHz}$, with peak protection of $15 \mathrm{~dB}$ at $15 \mathrm{kHz}$, the frequency suffering the largest threshold shift. At higher or lower frequencies TTSs in de-efferented ears and protection in efferent-intact ears both decreased. Noise at $40 \mathrm{~dB}$ SPL had no exacerbating effect on TTSs in de-efferented ears, and protection in efferent-intact ears was similar to that in group Silence 3 exposed to the loud tone in a background of silence. With noise at $60 \mathrm{~dB}$ SPL there was a significant increase in TTSs at high frequencies in de-efferented ears and a large increase in protection at these frequencies in efferent-intact ears. Mean protection of $>10 \mathrm{~dB}$ now occurred from 12 to $28 \mathrm{kHz}$, with peak protection of $23 \mathrm{~dB}$ at 19 $\mathrm{kHz}$. With noise at $80 \mathrm{~dB}$ SPL there was further exacerbation of TTSs in de-efferented ears, particularly at high frequencies, and more protection in efferent-intact ears. Mean protection of $>10 \mathrm{~dB}$ extended from 11 to $28 \mathrm{kHz}$ with peak protection of almost $30 \mathrm{~dB}$ from 20 to $22 \mathrm{kHz}$. 

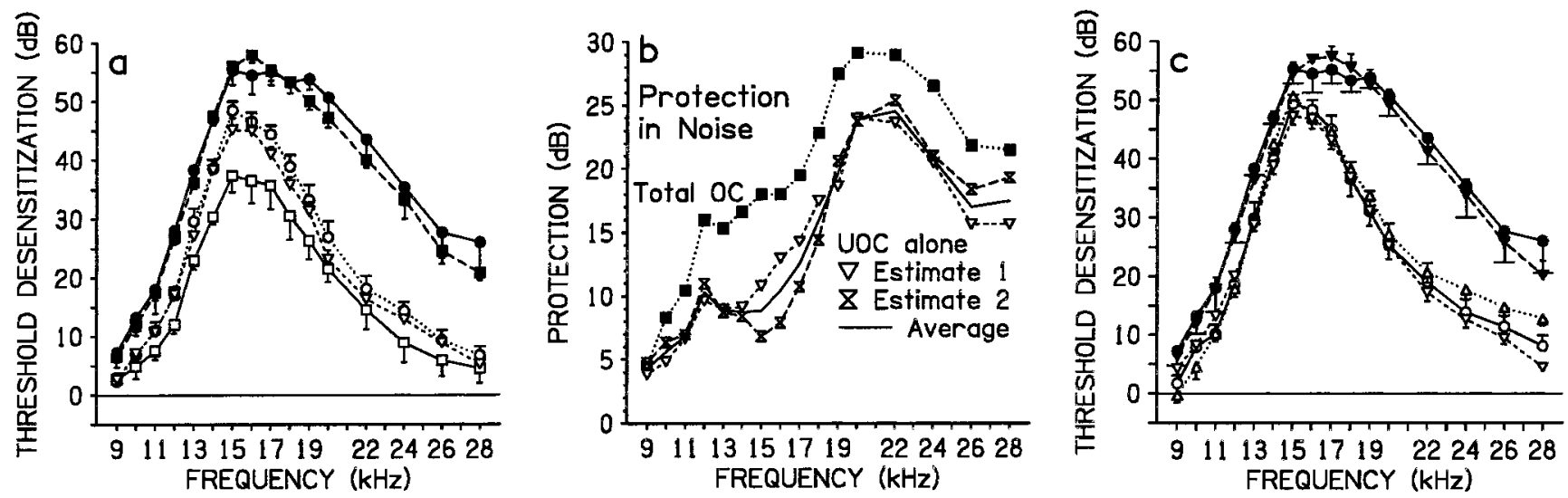

Figure 3. Protection by the uncrossed efferent pathways from loud tone-induced TTSs in a noise background can be elicited by monaural stimulation. $a$, $c$, TTSs in various groups after loud tone exposure either binaurally or monaurally, in a background of noise at $80 \mathrm{~dB}$ SPL or of silence. Data are group mean data (error bars indicate SE) from efferent-intact ears, totally de-efferented ears, or crossed de-efferented ears. $a$, Both crossed and uncrossed pathways are responsible for the cochlear protection seen with binaural tone exposure in binaural noise. In efferent-intact ears, binaural tone exposure in a noise background (Noise 3 efferent-intact ears, open squares and full line) produced low TTS. In totally de-efferented ears, the binaural tone exposure in noise (Noise 3 totally de-efferented ears, closed circles and full line; and Noise 5 totally de-efferented ears, closed squares and long dashed line) produced large TTS. In crossed de-efferented ears, the binaural tone exposure in noise (Noise 4 crossed de-efferented ears, open circles and dotted line; Noise 5 crossed de-efferented ears, open inverted triangles and short dashed line) produced an intermediate amount of TTSs at most frequencies. $b$, Protection of the cochlea in noise backgrounds by uncrossed efferent pathways (UOC alone) compared to that by action of both crossed and uncrossed efferent pathways $($ Total $O C)$. For the former, Estimate 1 was obtained from group Noise 5 from the difference between mean frequency-specific TTSs in the totally de-efferented ears and in the crossed de-efferented ears; Estimate 2 was the difference between mean data from the totally de-efferented ears in Noise 3 and the crossed de-efferented ears from Noise 4. The average (Average, full line) of these two estimates is also illustrated. Total OC protection is that previously illustrated in Figure $2 c$ for Noise 3 as the difference in mean frequency-specific TTSs in totally de-efferented ears and efferent-intact ears of the same group. $c$, Protection by uncrossed pathways can be elicited by monaural exposure to the loud tone in a monaural noise background (at $80 \mathrm{~dB}$ SPL). In totally de-efferented ears, monaural tone exposure in monaural noise (Noise 7 totally de-efferented ears, closed inverted triangles and long dashed line) produced large TTSs similar to that seen previously with binaural tone exposure in binaural noise (Noise 3 totally de-efferented ears, closed circles and full line). In efferent-intact ears, a noise background to a monaural tone exposure (Noise 6 efferent-intact ears, open circles and full line) did not exacerbate TTSs above that to a monaural tone exposure alone (Silence 1, open upright triangles, dotted line). Furthermore, in crossed de-efferented ears a noise background to a monaural tone exposure (Noise 7 crossed de-efferented ears, inverted triangles and long dashed line) also did not exacerbate TTSs above that to a monaural tone exposure alone (i.e., Silence 1), indicating that the intact uncrossed pathways in Noise 7 are able to protect from noise exacerbation of TTS.

\section{The enhanced cochlear protection to binaural tones in binaural noise backgrounds is attributable to activation of the uncrossed efferent pathways additional to the crossed pathways}

In confirmation of previous studies (Cody and Johnstone, 1982; Rajan and Johnstone, 1988; Rajan, 1995a,b), it was shown earlier that in a background of silence only the crossed efferent pathway mediates protection obtained with exposure to binaural loud sound. To determine if the enhanced protection in the higher-level noise backgrounds was also attributable to only this pathway, in group Noise 4 only this pathway was cut bilaterally (as in Silence 4). The loud tone and noise at $80 \mathrm{~dB}$ SPL were delivered binaurally. The identical treatment of the two ears in each animal resulted in bilaterally similar TTS, and data from corresponding frequencies in all animals were pooled. These data were compared (Fig. $3 a$ ) to data from efferent-intact ears and totally de-efferented ears in Noise 3 in which the binaural loud tone was also presented in binaural noise at $80 \mathrm{~dB}$ SPL. The comparison showed that crossed and uncrossed efferent pathways were involved in the enhanced protection in noise, in a frequency-dependent manner. Despite the absence of the crossed pathway in Noise 4, TTSs at all frequencies $(9-28 \mathrm{kHz})$ were still lower than in totally de-efferented ears of Noise 3 (Table $3 C$ ). Thus, in Noise 4 the intact uncrossed efferent pathways still significantly protected the cochlea over the entire affected frequency range. However, from 12 to $18 \mathrm{kHz}$, the crossed pathway provides significant protection in noise: in Noise 4, in which only this pathway was absent, these frequencies suffered significantly larger TTSs (Table $3 C$ ) than did Noise 3 efferent-intact ears which possessed crossed and uncrossed pathways.

The involvement of uncrossed pathways in the enhanced protection in noise was confirmed directly by a within-group test in group Noise 5. In this group crossed pathways were lesioned bilaterally, and then all efferent pathways (i.e., the remaining uncrossed pathways) to only one cochlea were lesioned. Then the cats were exposed binaurally to the loud tone in the background of noise at
$80 \mathrm{~dB}$ SPL. The ears that were only crossed de-efferented ears always suffered significantly lower TTSs (Fig. $3 a$, Table $3 C$ ) than the totally de-efferented ears; thus, in the crossed de-efferented ears, intact uncrossed pathways still protected the cochlea. (As shown in Fig. $3 a$, TTSs in totally de-efferented ears in Noise 5 were similar to those in totally de-efferented ears in Noise 3, and TTSs in crossed de-efferented Noise 5 ears were similar to those in crossed de-efferented Noise 4 ears. This is also demonstrated below in calculations of the protection provided by uncrossed efferent pathways.)

Two estimates were obtained of the protection provided by uncrossed efferent pathways in a background of noise at $80 \mathrm{~dB}$ SPL. A within-group estimate (Fig. 3b, UOC protection Estimate 1) was obtained from group Noise 5 from the difference between mean frequency-specific TTSs in totally de-efferented and crossed deefferented ears. An across-group estimate (Fig. 3b, UOC protection Estimate 2) was obtained from the difference between mean data from totally de-efferented ears in Noise 3 and crossed de-efferented ears in Noise 4. Protection by uncrossed pathways was biased toward higher frequencies (Fig. $3 b$ ): protection of $>10 \mathrm{~dB}$ was found consistently only at frequencies $>14 \mathrm{kHz}$ with peak protection of $\sim 24-25 \mathrm{~dB}$ occurring from 20 to $22 \mathrm{kHz}$ and declining to $\sim 17 \mathrm{~dB}$ at $28 \mathrm{kHz}$. Figure $3 b$ (Total $O C$ ) also compares this protection to that provided in noise when all efferent pathways were intact, calculated previously (Fig. 2c) from the difference between totally de-efferented and efferent-intact ears in group Noise 3. The pattern of protection in the latter case is dominated by the pattern caused by uncrossed pathways alone: protection of $>10 \mathrm{~dB}$ was consistently found from $11 \mathrm{kHz}$ to all higher frequencies. Peak protection of almost $30 \mathrm{~dB}$ was at $20-22 \mathrm{kHz}$, with a small decline to $\sim 22 \mathrm{~dB}$ at $28 \mathrm{kHz}$. This large protection in group Noise 3 was attributable to the involvement of both crossed and uncrossed efferent pathways (in efferent-intact ears in Noise 3) in the noise background. 


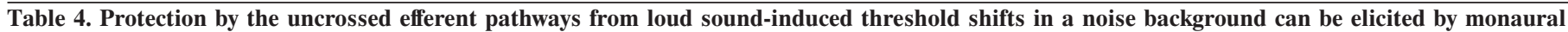
stimulation

\begin{tabular}{|c|c|c|c|}
\hline Comparison groups/treatments ${ }^{a}$ & $\begin{array}{l}\text { ANOVA } F \text { and } p \text { values (Inter- } \\
\text { action }=\text { group } \times \text { frequency in- } \\
\text { teraction) }\end{array}$ & $t$ test type ${ }^{b}$ and $\mathrm{df}^{c}$ & $\begin{array}{l}\text { Frequency range with significant } \\
\text { differences }{ }^{d} \text { and parameters of } \\
\text { significance }\end{array}$ \\
\hline
\end{tabular}

A. Cochlear protection from loud sound-induced TTS can be elicited by monaural loud sound in a monaural noise background

Silence $1(\mathrm{OC}+) \quad$ Noise $6(\mathrm{OC}+) \quad$ Group $F=0.185 ; p>0.6$

Monaural exposure $\quad$ Monaural exposure in noise $\quad$ Interaction $F=1.028 ; p=0.4$

\begin{tabular}{|c|c|c|c|c|}
\hline Noise $3 \mathrm{OC}-$ & Noise $6(\mathrm{OC}+)$ & Group $F=25.44 ; p<0.001$ & One-tail, df $=10$ & $\begin{array}{l}12-28 \mathrm{kHz} ; t \text { always }>2.06 ; p \\
\text { generally } \mathrm{p}<0.01\end{array}$ \\
\hline
\end{tabular}

Binaural exposure $\quad$ Monaural exposure in noise $\quad$ Interaction $F=6.022 ; p<0.001$

in noise

B. Cochlear protection from loud sound-induced TTS elicited in the monaural condition is attributable to only the uncrossed efferent pathways
Noise $6(\mathrm{OC}+) \quad$ Noise $7 \mathrm{OC}-$
Group $F=42.22 ; p<0.001$
One-tail, df $=9$
9-28 kHz; $t$ always $>2.65 ; p$ generally $<0.005$

Monaural exposure

Monaural exposure in noise

Interaction $F=8.51 ; p<0.001$

in noise

\begin{abstract}
Noise 7 COC- $\quad$ Noise 7 OC-
Group $F=111.7 ; p<0.001$

One-tail, $\mathrm{df}=8$

$10-28 \mathrm{kHz} ; t$ always $>2.43 ; p$

Monaural exposure

Monaural exposure in noise

Interaction $F=12.34 ; p<0.001$

in noise

$\begin{array}{lll}\text { Noise } 6(\mathrm{OC}+) & \text { Noise } 7 \mathrm{COC}- & \text { Group } F=0.068 ; p=0.8 \\ \begin{array}{l}\text { Monaural exposure } \\ \text { in noise }\end{array} & \text { Monaural exposure in noise } & \text { Interaction } F=0.53 ; p=0.9\end{array}$

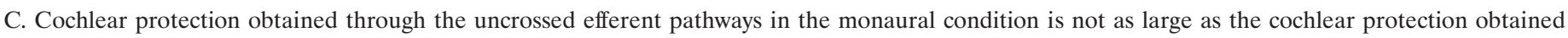
through crossed and uncrossed efferent pathways in the binaural condition
Noise 7 COC-
Noise $3 \mathrm{OC}+$
Group $F=6.57 ; p<0.05$
One-tail, df $=9$
11-16 kHz; $t$ always $>2.29 ; p$ generally $<0.01$
in noise
Noise $7 \mathrm{COC}-\quad$ Noise $4(\mathrm{COC}-) \quad$ Group $F=0.018 ; p=0.9$
Monaural exposure $\quad$ Binaural exposure in noise $\quad$ Interaction $F=0.58 ; p=0.9$
in noise

Monaural exposure $\quad$ Binaural exposure in noise $\quad$ Interaction $F=1.33 ; p=0.18$

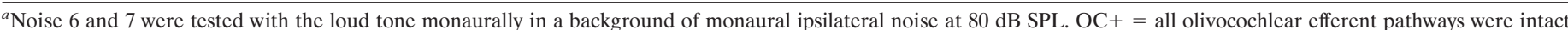

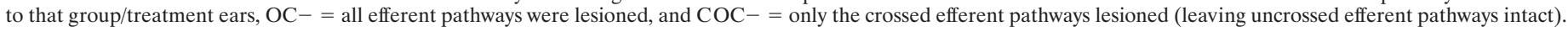

${ }^{b} t$ tests were performed when the ANOVA indicated significant differences.

${ }^{c}$ Degrees of freedom.

${ }^{d}$ Frequencies for which $t$ values were significant at $\alpha=0.05$ (i.e., $p$ at least $<0.05$ ).
\end{abstract}

\section{Protection by the uncrossed efferent pathways from loud sound-induced TTSs in a noise background can be elicited by monaural stimulation}

As noted above, in a background of silence binaural loud tones are required to activate protection, which is then mediated only by the crossed efferent pathway. I determined whether the (larger) efferent protection obtained in a background of noise, through involvement of both crossed and uncrossed pathways, also required binaural stimulation. In two groups tests were done with only monaural loud sound in monaural ipsilateral noise at $80 \mathrm{~dB}$ SPL. In group Noise 6, all efferent pathways were intact, and TTSs (Fig. 3c) was not significantly different (Table $4 A$ ) from TTSs in group Silence 1 in which the monaural loud tone was presented in a background of silence. In contrast, as also illustrated in Figure $3 c$, after total de-efferentation (in Noise 3; totally de-efferented ears) TTSs to the monaural tone in the monaural noise background was greater than TTSs in group Silence 1 to the monaural loud tone in a background of silence. (Although loud tone and noise were applied binaurally in Noise 3 , the total de-efferentation of ears for which data are considered here effectively renders those ears monaural.) Comparison of Noise 6 data against those from totally de-efferented ears of Noise 3 showed that TTSs from 12 to $28 \mathrm{kHz}$ in Noise 6 were significantly lower than in Noise 3 totally de- efferented ears (Table $4 A$ ). This confirmed that in the monaural condition of Noise 6 , intact efferent pathways prevented noise from exacerbating tone-induced TTS.

To determine which efferent component was responsible for this monaurally elicited protection, in group noise 7 two lesions were placed, as in Noise 5; one to cut the crossed pathways bilaterally, and the second to cut all efferent pathways (i.e., the remaining uncrossed pathways) to only one cochlea. The monaural loud tone and noise were first applied in the crossed de-efferented ears and later in the totally de-efferented ears.

Threshold shifts in the crossed de-efferented Noise 7 ears were similar to those in the efferent-intact ears of Noise 6 (Fig. 3c, Table $3 B$ ). However, in the totally de-efferented ears in Noise 7, TTSs at $10-28 \mathrm{kHz}$ were significantly higher than in efferent-intact ears of Noise 6 (Fig. 3c, Table $4 B$ ) and also significantly higher than in crossed de-efferented Noise 7 ears (Fig. $3 c$, Table 4B). Thus, under monaural conditions, uncrossed efferent pathways can act alone to prevent noise exacerbating tone-induced damage. However, at some frequencies, this protection is not as great as that obtained in the (binaural) condition in which crossed and uncrossed efferent pathways are active in protection. Crossed de-efferented monaurally exposed Noise 7 ears (intact uncrossed pathways) suffered significantly greater TTSs from 11 to $16 \mathrm{kHz}$ than did the efferent- 
intact binaurally exposed ears in group Noise 3 (Table $4 C$ ). It has been shown earlier that in the conditions in these Noise 3 ears (all efferents intact, and tested binaurally), both crossed and uncrossed pathways are active in protection. The extra protection in these ears compared to the crossed de-efferented Noise 7 ears was attributable to the action of the crossed pathways in the Noise 3 ears.

These analyses convincingly demonstrate that the protection in the binaural tone and noise condition was caused by both crossed and uncrossed efferent pathways. A further analysis refuted the alternative hypothesis that stronger activity in uncrossed efferents was responsible for the larger protection in the binaural condition compared to the monaural condition. Comparison of TTSs in crossed de-efferented monaurally exposed Noise 7 ears against those in crossed de-efferented binaurally exposed group Noise 4 found no significant differences (Table $4 C$ ) confirming that uncrossed efferent pathways (intact in both groups) are as effectively activated by the monaural test condition as by the binaural test condition.

In contrast, the results from the monaurally tested groups reconfirmed that binaural conditions are required for the crossed efferent pathway to be activated, even in a noise background. In this background the monaural loud tone produced similar TTSs whether the crossed pathway was intact (Noise 6) or lesioned (Noise 7), as long as uncrossed pathways were intact. Thus, in a background of noise (as in a background of silence, as shown earlier) the crossed efferent pathway is not activated by a monaural loud tone. In accord with this conclusion, the monaural loud tone produced similar TTSs whether applied in a background of noise (Noise 6) or in a background of silence (Silence 1).

\section{DISCUSSION}

This report establishes three important results. First, in the absence of functional cochlear efferent pathways even a moderate noise background, itself not causing any damage, significantly exacerbates damaging effects of a loud tone, particularly at higher frequencies of the tone-affected range. Second, in noise backgrounds there is activation of efferent pathways additional to those activated by a binaural loud tone in a background of silence, and this enhances cochlear protection compared to that in silence. Additional efferent pathways activated in noise are uncrossed efferent pathways not activated by the loud tone alone. Finally, in noise, uncrossed efferents are "automatically" activated by loud tones, even under monaural conditions of loud tone and noise, and prevent noise from exacerbating tone-induced damage. The protective effects of uncrossed pathways are only revealed when they are inactivated.

The observation that uncrossed efferent pathways have cochlear effects in a noise background is consistent with the demonstration (Brown et al., 1998) that single uncrossed efferent neurons are driven more effectively by noise than tones, and the observation (Kawase and Liberman, 1993; Kawase et al., 1993) of other (antimasking) cochlear effects of uncrossed pathways in noise. In the latter case, when responses to tones in one ear are masked by noise in that ear, addition of noise to the other ear activates uncrossed pathways to the test ear to reduce some of the masking. The laterality of noise application to reveal uncrossed efferent antimasking effects and the protection of the present study differ in that protection is observed when noise and tone are presented to the same ear alone. This may only reflect the difference that in studies of anti-masking effects, tone levels likely to damage hearing were not used. This difference may also account for the finding, in single-unit studies, that uncrossed efferent neurons are driven by the ear other than the one to which they project (Liberman and Brown, 1986) and facilitated by noise in the projection ear (Liberman, 1988; Brown et al., 1998), whereas the present study shows that uncrossed efferents to a cochlea protect when noise and loud tone are in their projection ear alone (i.e., monaural loud tone and noise) and binaural tone in noise did not produce any more uncrossed efferent protection. (The extra protection in the binaural condition was attributable to activation of the crossed efferent pathway by binaural tones, not to any greater activation of uncrossed pathways.) In the single-unit studies, too, sounds likely to cause damage to hearing sensitivity were not used.

White noise did not cause TTSs; rather, in de-efferented ears it exacerbated damage caused by the loud tone, particularly at high frequencies in the affected range. Three features suggest that uncrossed efferents prevent this exacerbation rather than acting primarily on the mechanism whereby loud sound damages hearing sensitivity, whereas crossed efferents act specifically on the latter mechanism.

First, TTSs after a monaural loud tone in a noise background were identical to TTSs after the monaural tone in a background of silence. In the first condition only uncrossed efferents acted (to prevent noise from exacerbating loud tone-induced TTSs), whereas in the second, TTSs were not modulated by any efferent influence. Thus, when only uncrossed pathways protected, they prevented noise from exacerbating TTSs beyond levels caused by the loud tone. In contrast, when only the crossed pathway protected (with application of binaural loud tone in a background of silence), TTSs were reduced below the TTSs levels caused by the tone alone (monaural loud tone in a background of silence). Second, TTSs with application of binaural loud tone in background of noise were not lower than the protected levels seen with application of binaural loud tone in silence. In the first condition both crossed and uncrossed efferent pathways protected, whereas in the second only the crossed pathway protected. Thus, the additional activation of the uncrossed pathways in the first condition did not reduce TTSs below levels to which it had been reduced by the crossed pathway. Finally, uncrossed efferent protection (in background of noise) was greatest at higher frequencies at which noise most exacerbated (in de-efferented ears) TTSs. In all tests peak TTSs was focused at 15 $\mathrm{kHz}$ (although in noise backgrounds this peak broadened to include higher frequencies). However, protection at $15 \mathrm{kHz}$ was generally similar whether the binaural tone was applied in a background of silence, when only the crossed pathway protected, or in a noise background, when crossed and uncrossed efferents protected. This was not caused by saturation of uncrossed efferent protection: in contrast to protection at $15 \mathrm{kHz}$, much greater protection was obtained in a noise background at the higher frequencies at which noise exacerbated TTSs. (Note that even then TTSs at the higher frequencies did not significantly exceed that at $15 \mathrm{kHz}$ ). In contrast, crossed efferent protection was maximal at the frequency most affected by the tone alone, as also found previously (Rajan and Johnstone, 1988; Rajan, 1992, 1995a,b, 1996) using a larger range of loud tone levels and frequencies, in a background of silence.

These effects suggest strongly that in the overall TTSs caused when a tone occurs in a noisy background, the crossed pathway acts on the mechanism whereby loud tones cause TTSs, whereas the uncrossed pathway acts on the mechanism whereby noise, in deefferented ears, allows loud tones to cause more TTSs. Although the mechanism underlying TTSs is unknown, a current view (Patuzzi et al., 1989; Patuzzi, 1992) suggests it is caused by temporary inactivation of mechanosensitive transduction ion channels in OHCs. It has been suggested (Patuzzi and Rajan, 1990) that such inactivation occurs only for movement of the cochlear partition in one direction during sound-induced vibration of the partition. Noise may exacerbate loud tone-induced TTSs by biasing the cochlear partition further or for longer in this direction.

The two efferent pathways also differed in how they were activated to produce protection. Previous studies with loud tones, in a background of silence, have shown that the protective crossed efferent pathway is activated only by binaural stimulation. [Note that the input does not have to be binaurally equally intense (Cody and Johnstone, 1982; Rajan and Johnstone, 1988), a condition that translates credibly to the free-field in which loud sounds away from 
the midline will be unequally loud bilaterally (Rajan, 1995b).] This study extends that feature to noise backgrounds by showing that in a noise background, a monaural loud tone evoked protection only through uncrossed pathways. These various data suggest that protective crossed efferent neurons are activated frequency-specifically only by binaural tonal input. This is consistent with the report, when testing with binaural loud sounds in a background of silence, that protection requires binaural tones to be at the same frequency (Cody and Johnstone, 1982). In contrast, uncrossed pathways only protect from TTSs caused by tones in the presence of noise, but such activation occurs with monaural input from both tone and noise, resulting in expression of uncrossed efferent protection as fully as with binaural testing. These data suggest that crossed efferent neurons involved in protection do not require convergence of inputs from different frequency channels but require binaural input for expression of cochlear protection. In contrast, uncrossed efferent neurons involved in protection appear to require convergence of inputs from many frequency channels, but this convergence occurs for monaural input from the ear to which they project (and, ultimately, protect).

The crossed (contralateral) efferent pathway consists almost exclusively of MOCS neurons terminating on OHCs (Guinan et al., 1983; Warr et al., 1986); protective effects of the crossed pathway must therefore be exercised only at this cochlear site. The uncrossed (ipsilateral) pathway consists of LOCS neurons terminating on dendrites of cochlear afferent neurons, and MOCS neurons terminating on OHCs (Guinan et al., 1983; Warr et al., 1986). Potentially either subcomponent could be responsible for uncrossed pathway protection. It is generally held (Weiderhold, 1986; Guinan, 1988) that all efferent effects at the cochlea to date, and responses of single efferents, are attributable solely to MOCS neurons terminating on OHCs. It therefore seems most parsimonious to assume that uncrossed efferent protection is attributable only to (ipsilateral) MOCS neurons (i.e., the UMOCS); also it is difficult to see how actions at dendrites of cochlear afferent neurons could prevent noise from exacerbating loud tone-induced damage.

Finally, the present results have a clear and important relevance to human susceptibility to loud sound-induced damage to cochlear hearing sensitivity. Exposure to loud sounds very often would occur in environments with an ambient level of noise (such as workplace or recreational environments). The present study shows that uncrossed cochlear efferents are already "primed" to be activated in such environments to prevent noise from exacerbating damage caused by any loud sounds. Dysfunction of efferents, particularly uncrossed efferents, could potentiate damage to hearing sensitivity and may be responsible for some effects seen in cases of occupational hearing damage, an increasing epidemiological problem. Designing conditions to optimize the action of efferent pathways in noisy environments that carry a risk of exposure to loud sound could significantly ameliorate cochlear damage caused by loud sound in such environments.

\section{REFERENCES}

Borg E, Canlon B, Engstrom B (1995) Noise-induced hearing loss: literature review and experiments in rabbits. Scand Audiol [Suppl]40:1-59.

Brown MC, Kujawa SG, Duca ML (1998) Single olivocochlear neurons in the guinea pig. I. Binaural facilitation of responses to high-level noise. J Neurophysiol 79:3077-3087.

Cody AR (1992) Acoustic lesions in the mammalian cochlea: implications for the spatial distribution of the "active process." Hear Res 62:166-172.

Cody AR, Johnstone BM (1982) Temporary threshold shift modified by binaural acoustic stimulation. Hear Res 6:199-205.

Cody AR, Russell IJ (1986) The response of mammalian cochlear hair cells to acoustic overstimulation. In: Basic and applied aspects of noiseinduced hearing loss (Salvi RJ, Henderson D, Hamernik RP, Colletti V, eds), pp 149-161. New York: Plenum.

Cooper NP, Rhode WS (1992) Basilar membrane mechanics in the hook region of cat and guinea-pig cochleae: sharp tuning and non-linearity in the absence of baseline position shifts. Hear Res 63:163-190.
Dallos P, Harris D (1978) Properties of auditory nerve responses in absence of outer hair cells. J Neurophysiol 41:365-383.

Dew LA, Owen Jr RG, Mulroy MJ (1993) Changes in the size and shape of auditory hair cells in vivo during noise-induced temporary threshold shift. Hear Res 66:99-107.

Galambos R (1956) Suppression of auditory nerve activity by stimulation of efferent fibres to the cochlea. J Neurophysiol 19:424-437.

Gao W-y, Ding D-1, Zheng X-y, Ruan F-m, Liu Y-j (1992) A comparison of changes in the stereocilia between temporary and permanent hearing losses in acoustic trauma. Hear Res 62:27-41.

Guinan Jr JJ (1988) Physiology of the olivocochlear efferents. In: Auditory pathway: structure and function (Syka J, Masterton RB, eds), pp 253-267. New York: Plenum.

Guinan JJ Jr, Warr WB, Norris BE (1983) Differential olivocochlear projections from lateral versus medial zones of the superior olivary complex. J Comp Neurol 221:358-370.

Handrock M, Zeisberg J (1982) The influence of the efferent system on adaptation, temporary and permanent threshold shift. Arch Otorhinolaryngol 234:191-195.

Harding GW, Baggot PJ, Bohne BA (1992) Height changes in the organ of Corti after noise exposure. Hear Res 63:26-36.

Harrison RV, Evans EF (1977) The effects of hair cell loss (restricted to outer hair cells) on the threshold and tuning properties of cochlear fibres in the guinea pig. Inner Ear Biol 68:105-124.

Kawase T, Liberman MC (1993) Antimasking effects of the olivocochlear reflex. I. Enhancement of compound action potentials to masked tones. J Neurophysiol 70:2519-2532.

Kawase T, Delgutte B, Liberman MC (1993) Antimasking effects of the olivocochlear reflex. II. Enhancement of auditory-nerve response to masked tones. J Neurophysiol 70:2533-2549.

Kujawa SG, Liberman MC (1997) Conditioning-related protection from acoustic injury: effects of chronic deefferentation and sham surgery. J Neurophysiol 78:3095-3106.

Liberman MC (1988) Response properties of cochlear efferent neurons: monaural vs binaural stimulation and the effects of noise. J Neurophysiol 60:1779-1798.

Liberman MC, Brown MC (1986) Physiology and anatomy of single olivocochlear neurons in the cat. Hear Res 24:17-36.

Liberman MC, Dodds LW (1987) Acute ultrastructural changes in acoustic trauma: serial-section reconstruction of stereocilia and cuticular plates. Hear Res 26:45-64.

Liberman MC, Gao WY (1995) Chronic cochlear deefferentation and susceptibility to permanent acoustic injury. Hear Res 90:158-168.

Liberman MC, Kiang NYS (1978) Acoustic trauma in cats. Acta Octolaryngol [Suppl] 358:1-63.

Liberman MC, Dodds LW, Learson DA (1986) Structure-function correlation in noise-damaged ears: a light and electron-microscopic study. In: Basic and applied aspects of noise-induced hearing loss (Salvi RJ, Henderson D, Hamernik RP, Colletti V, eds), pp 163-177. New York: Plenum.

Patuzzi R (1992) Effect of noise on auditory nerve responses. In: Noiseinduced hearing loss (Dancer AL, Henderson D, Salvi RJ, Hamernik RP, eds), pp 45-59. St. Louis: Mosby.

Patuzzi R, Rajan R (1990) Does electrical stimulation of the crossed olivo-cochlear bundle produce movement of the organ of Corti? Hear Res 45:15-32.

Patuzzi R, Robertson D (1988) Tuning in the mammalian cochlea. Physiol Rev 68:1009-1082.

Patuzzi R, Yates GK, Johnstone BM (1989) Outer hair cell receptor current and sensorineural hearing loss. Hear Res 42:47-72.

Rajan R (1992) Protective functions of the efferent pathways to the mammalian cochlea: a review. In: Noise-induced hearing loss (Dancer AL, Henderson D, Salvi RJ, and Hamernik RP, eds), pp 45-59. St. Louis: Mosby.

Rajan R (1995a) Involvement of cochlear efferent pathways in protective effects elicited with binaural loud sound exposure in cats. J Neurophysiol 74:582-597.

Rajan R (1995b) Frequency and loss dependence of the protective effects of the olivocochlear pathways in cats. J Neurophysiol 74:598-615.

Rajan R (1996) Additivity of loud-sound-induced threshold losses in the cat under conditions of active or inactive cochlear efferent-mediated protection. J Neurophysiol 75:1601-1618.

Rajan R, Johnstone BM (1988) Binaural acoustic stimulation exercises protective effects at the cochlea that mimic the effects of electrical stimulation of an auditory efferent pathway. Brain Res 459:241-255.

Rajan R, Irvine DRF, Cassell JF (1991) Normative N1 audiogram data for the barbiturate-anaesthetised domestic cat. Hear Res 53:153-158.

Robertson D (1982) Effects of acoustic trauma on stereocilia structure and spiral ganglion tuning properties in the guinea pig cochlea. Hear Res 7:55-74.

Robertson D, Johnstone BM (1979) Aberrant tonotopic organization in the inner ear damaged by kanamycin. J Acoust Soc Am 66:466-469.

Robertson D, Johnstone BM (1980) Acoustic trauma in the guinea pig cochlea: early changes in ultrastructure and neural threshold. Hear Res 3:167-179.

Robertson D, Johnstone BM, McGill TJ (1980) Effects of loud tones on 
the inner ear: a combined electrophysiological and ultrastructural study. Hear Res 2:39-53.

Ruggero MA, Rich NC, Recio A (1993) Alteration of basilar membrane responses to sound by acoustic overstimulation. In: Biophysics of hair cell sensory systems (Duifhuis H, Horst JW, van Dijk P, van Netten SM, eds), pp 258-265. Singapore: World Scientific.

Schmeidt RA, Zwislocki JJ, Hamernik RP (1980) Effects of hair cell lesions on responses of cochlear nerve fibres. I. Lesions, tuning curves, two-tone inhibition, and responses to trapezoidal wave forms. J Neurophysiol 43:1367-1389.

Tilney LG, Saunders JC, Egelman E, DeRosier DJ (1982) Changes in the organization of actin filaments in the stereocilia of noise-damaged lizard cochleae. Hear Res 7:181-197.

Warr WB, Guinan Jr JJ, White JS (1986) Organization of the efferent fibres. In: Neurobiology of hearing: the cochlea (Altschuler RA, Hoffman DW, Bobbin RP, eds), pp 333-348. New York: Raven.

Warren III, EH, Liberman MC (1989) Effects of contralateral sound on auditory-nerve responses. I. Contributions of cochlear efferents. Hear Res 37:89-104.

Weiderhold ML (1986) Physiology of the olivocochlear system. In: Neurobiology of hearing: the cochlea (Altschuler RA, Hoffman DW, Bobbin RP, eds), pp 349-370. New York: Raven.

Zheng X-Y, Henderson D, McFadden SL, Hu B-H (1997a) The role of the cochlear efferent system in acquired resistance to noise-induced hearing loss. Hear Res 104:191-203.

Zheng X-Y, Henderson D, Hu B-H, Ding D-L, McFadden SL (1997b)

The influence of the cochlear efferent system on chronic acoustic trauma. Hear Res 107:147-159. 